\title{
The Influence of Perfectionism and Thought Suppression on Negative Rumination*
}

\author{
Kenji Shimizu \\ Shinshu University, Nagano, Japan
}

\author{
Hisayo Shimizu \\ Hiroshima University, Hiroshima, Japan
}

\begin{abstract}
Major depressive disorder is known to show symptoms of emotional and physical disturbance. One risk factor that aggravates depressive symptoms is negative rumination. Perfectionism has been recognized as a personality trait that has maladaptive and adaptive aspects relative to depression. Perfectionism also bears certain relations to negative rumination. The present study aims to clarify the mechanism by which a perfectionist increases his or her intensity of negative rumination while revealing the types of situation he/she might act on and the style of coping he/she might adopt. We conducted a panel survey with an interval period of four weeks. Self-reported questionnaires to assess negative rumination (covering the T1-T2 interval), perfectionistic cognition ( $\mathrm{T} 1$ only), thought suppression ( $\mathrm{T} 1$ only), and stressors faced in daily life (T2 only) were administered to 272 non-clinical university students. The findings are as follows: When perfectionists are engaged in stressful situations in a compulsive manner, they achieve the opposite result of what they intended due to their compulsive way of enthusiastically engaging in thought suppression. It is suggested that an effective treatment for perfectionists to escape from negative rumination would set them free from endless attempts to suppress their negative thoughts.
\end{abstract}

Keywords: perfectionism, negative rumination, thought suppression

\section{Introduction}

Major depressive disorder is known to be accompanied by symptoms of emotional and physical disturbance. The typical dysfunction in emotional state is mood stagnation and that in the physical state is the deterioration of appetite, weight loss, and changes in sleeping patterns. Depression easily becomes chronic, presenting higher levels of morbidity and the risk of suicide. It is recognized that depression is a major problem in our society. It is important to investigate causal factors for the onset and chronicity of depression. In this study, we investigated an interconnection among variables that are assumed to be casual factors of depression and tried to clarify relations of causality underlying pathological mechanisms.

One of the risk factors known to aggravate depressive symptoms is negative rumination. Negative rumination is defined as a response style of depressed mood characterized by repetitive thinking about one's own symptoms, their possible causes, and consequences (Nolen-Hoeksema, 1991). Negative rumination

\footnotetext{
*Acknowledgements: The authors make a grateful acknowledgement to Professor Akira Imai, Faculty of Arts, Shinshu University in drafting this manuscript. This work was supported by JSPS KAKENHI (Grants-in-Aid for Scientific Research) Grant Number 23730652.

Kenji Shimizu, Ph.D., Associate professor, Faculty of Arts, Shinshu University.

Hisayo Shimizu, Ph.D., Associate professor, Department of Education, Hiroshima University.
} 
provides psychological distress due to repetitive thoughts about previous aversive events relating to one's self, and it is not easy to end such continuous negative thinking patterns (Ito, Takenaka, \& Agari, 2001). Therefore, the manner in which we can prevent this rumination chain is a topic of concern. What personality traits enhance rumination?

Perfectionism has been recognized as one personality trait with maladaptive and adaptive aspects relative to depression (Hamachek, 1978). The tendency to demand that one performs perfectly is called self-oriented perfectionism (Hewitt \& Flett, 1991). This construct has been conceived as a relatively stable self-schema among individuals. Because the desire for perfection raises a personal motivation to varying levels, the desire may produce better performance and excellent results. However, one may not be always satisfied with the success one has, resulting in an increase in frustration and causing a stereotyped behavior in daily life. Thus, perfectionists are vulnerable to various forms of psychological distress, anxiety, and negative rumination in depression.

Perfectionism shows adaptive and maladaptive aspects in social situations. One adaptive aspect is having personal standards (PS), otherwise known as "perfectionistic strivings", as a representative trait of self-oriented perfectionism (Kobori \& Tanno, 2004; Schiena, Lumine, Philippot, \& Douilliez, 2012). Perfectionistic strivings are assumed to form cognitive structures to set higher goals for oneself and are regarded as the adaptive component of perfectionism. The maladaptive aspect is related to the concern over making mistakes (CM), otherwise known as "perfectionistic concerns". The perfectionistic concerns are cognitive structures that make one's mind full of fears of failure and criticism from others; this is why perfectionistic concerns are maladaptive. Perfectionism is considered to have a certain association with depressive symptoms and feelings. CM shows an especially positive correlation with the development of depressive symptoms in clinical populations (Enns \& Cox, 2002) as well as in non-clinical student populations (Minarik \& Ahrens, 1996). Schiena, Lumine, Philippot, and Douilliez (2012) also demonstrated that negative rumination plays a role in mediating between $\mathrm{CM}$ and depressive symptoms. As mentioned above, perfectionism involves the development of depressive symptoms and mental illness among clinical and non-clinical populations.

It has been revealed that coping strategies for stressful situations exert a remarkable influence on the increase and decrease of negative rumination. In particular, thought suppression is shown to result in the aggravation of negative rumination and depressive symptoms (Aldao, Nolen-Hoeksema, \& Schweizer, 2010). Thought suppression is defined as a state in which one avoids experiencing a negative emotional reaction and strives to inhibit thoughts with hate-involving content (Wegner, 1989). In spite of one's own coping efforts, thought suppression frequently brings the maladaptive consequences of anxiety and fear. For example, while one may attempt to suppress a strange thought that easily re-appears, despite one's efforts at inhibition (Wegner, Schneider, Carter, \& White, 1987). This recursive process causes a "muddy stream" of negative thoughts and is known as the "paradoxical effect of suppression". As a result, regardless of individual coping efforts, the recurring nature of thought suppression results in unpleasant thought and more serious depressive feelings and symptoms follow.

The present study aims to clarify the mechanism by which a perfectionist increases his or her intensity of negative rumination with a focus on revealing the types of situation he/she might act on and the style of coping he/she might engage in. If we can find clues to prevent negative rumination, this will aid us in maintaining a healthy life. 


\section{Materials and Methods}

\section{Participants}

Participants were 272 undergraduate students who studied a certain psychology course at a university $(M=$ 18.6 years, $S D=1.01$ ). The current study was conducted as a panel survey. Self-report scale for negative rumination was applied at Time-1 (T1) and Time-2 (T2) to product difference score for an analysis of changes over time between T1 and T2. Scales of perfectionism and thought suppression were administered at T1 only, because these two scores were assumed to be stable, and not change over time. Daily hassles as a stressor scale was only used at $\mathrm{T} 2$. The stressor scale score was estimated retrospectively in the last four weeks. The interval of T2 was four weeks from T1.

We concentrated on the difference in the negative rumination score from $\mathrm{T} 1$ to $\mathrm{T} 2$. The most important point was how large and up to what extent personality factors such as perfectionism, coping strategies (thought suppression), and stressful situations (daily hassles) might contribute to and predict the difference in negative rumination score from $\mathrm{T} 1$ to $\mathrm{T} 2$.

\section{NRS (Negative Rumination Scale)}

The NRS score (Ito et al., 2001) consists of 11-item measurement scales. This is to measure how often participants experience negative and repetitive thoughts in their daily lives (e.g., I tend to repeatedly reflect on unpleasant thoughts with the same content). Each item was evaluated on a 5-point Likert scale, ranging from "Strongly disagree (1)" to "Strongly agree (5)". The score was collected at T1 and again at T2.

\section{MPCI (Multidimensional Perfectionism Cognition Inventory)}

The MPCI (Kobori \& Tanno, 2004) is a 15-item measurement scale. It consists of the following three factors - personal standards (e.g., It is important to set a high standard for oneself), concern over mistakes (e.g., If I fail, my self-value will fall), and pursuit of perfection (e.g., I must be perfect). In this study, we applied two factors - personal standards (typically regarded as an adaptive aspect of perfectionism) and CM (typically regarded as a maladaptive aspect). Each item was evaluated on a 5-point Likert scale, ranging from "Not at all (1)" to "Always (5)". The instruction was as follows: "Please answer how often a thought or a similar thought appeared in your head during the last week". This scale was completed only at T1.

\section{Thought Suppression Scale}

Thought suppression is scored by a 6-item measurement scale (Matsumoto, 2008) to estimate how individuals cope when strange thoughts appear in their heads. We used the Japanese version of WBSI (the White Bear Suppression Inventory) (Wegner \& Zanakos, 1994). Using the Japanese version of the WBSI, items regarding concrete coping strategies for one's thoughts were used (e.g., I always try to put problems out of mind). Each item was evaluated on a 5-point Likert scale, ranging from "Not at all (1)" to "Always (5)". This scale was completed only at $\mathrm{T} 1$.

\section{Stressor Scale of Daily Hassles for Undergraduate Students}

Stressors associated with daily hassles are scored using a 23-item measurement scale to estimate the frequency at which an individual encounters "daily hassle" events among Japanese undergraduates (Shima, 1992; 1999). It evaluates the level of daily irritation for a student's life (e.g., There is a person who hates me). The instructions proceed as follows: "There is a description of being irritated in daily life. Did you experience the following events during the last approximately four weeks"? Each item was scored on a 5-point Likert scale, 
ranging from "I did not experience and feel it at all (0)" to "I was worried about it very much (4)". This scale was completed only at $\mathrm{T} 2$.

\section{Results}

\section{Descriptive Statistics and Cross Correlation Coefficients}

Descriptive statistics and zero-order cross-correlations among scores obtained are shown in Table 1. Scores of PS and CM indicate weak positive correlation $(r=0.30)$. These scores are assumed to be different in conceptual meaning and adaptive contents, but it is also asserted that both scores have a certain interrelationship due to the similarity of perfectionistic cognition. This positive correlation of 0.30 was similar to the results obtained by Kobori and Tanno (2004) and was consistent with their findings. PS shows a null correlation with thought suppression, stressors, and negative rumination. On the other hand, CM indicates positively weak to moderate correlations between thought suppression, stressors, and negative rumination. Becoming sensitive to making a mistake is positively related to performing maladaptive coping strategies, creating irritating situations, and falling into repetitive thinking loops to some extent. However, the cognitive trait of setting a high goal measured as a personal standard seems to not have a clear connection to thought suppression, stressors, and negative rumination.

Table1

Descriptive Statistics and Zero-Order Correlations for Personal Standard, Concern for Mistakes, Thought Suppression, Stressor, and Negative Rumination $(T 1 \& T 2)$

\begin{tabular}{|c|c|c|c|c|c|c|c|c|c|}
\hline & Variables & $\begin{array}{l}\text { Personal } \\
\text { standards }\end{array}$ & $\begin{array}{l}\text { Concern } \\
\text { over } \\
\text { mistakes }\end{array}$ & $\begin{array}{l}\text { Thought } \\
\text { suppression }\end{array}$ & $\begin{array}{l}\text { Daily } \\
\text { hassles } \\
\text { (Stressor) }\end{array}$ & $\begin{array}{l}\text { Negative } \\
\text { rumination } \\
\text { (Time 1) }\end{array}$ & Mean & $S D$ & $\alpha$ \\
\hline 1 & Personal standards & & & & & & 16.61 & 4.86 & 0.88 \\
\hline 2 & Concern over mistakes & $0.30^{*}$ & & & & & 16.52 & 4.18 & 0.76 \\
\hline 3 & Thought suppression & 0.07 & $0.37^{*}$ & & & & 17.02 & 5.46 & 0.79 \\
\hline 4 & Daily hassles(Stressor) & -0.05 & $0.29^{*}$ & $0.34^{*}$ & & & 33.09 & 15.57 & 0.90 \\
\hline 5 & Negative rumination (Time1) & -0.08 & $0.44^{*}$ & $0.54^{*}$ & $0.35^{*}$ & & 32.42 & 9.24 & 0.89 \\
\hline 6 & Negative rumination (Time2) & -0.03 & $0.40^{*}$ & $0.50^{*}$ & $0.46^{*}$ & $0.76^{*}$ & 31.16 & 8.94 & 0.89 \\
\hline
\end{tabular}

Note. ${ }^{*} p<0.05, n=272$.

\section{Hierarchical Multiple Regression Analysis}

A hierarchical multiple regression analysis was performed, with negative rumination score (T2) as a criterion variable. Predictors were mean-centered and gender difference was entered as a control variable, with negative rumination (T1) as a covariate in Step 1. Main effects of PS (or CM), thought suppression, and stressors were entered in Step 2, two-way interaction effects in Step 3, and a three-way interaction effect in Step 4. Simple regression equations were used to investigate further significant interaction effects. Referring to J. Cohen and P. Cohen (1983) and Aiken and West (1991), a simple slope was calculated for a significant three-way interaction effect (using $\pm 1 S D, 1 S D=$ high, $-1 S D=$ low, respectively).

\section{PS (Personal Standards)}

The results of the hierarchical multiple regression analysis concerning PS showed a significant main effect for stressors $(\beta=0.113, p<0.05)$ and a three-way interaction effect with stressors, thought suppression, and PS $(\beta=-0.002, p<0.05)$. These results are shown in Table 2 . 
Table 2

Hierarchical Multiple Regression Analysis of Negative Rumination (Time 2) on PS Thought Suppresion and Stressor

\begin{tabular}{lcccc}
\hline & Step 1 & Step 2 & Step 3 & Step 4 \\
\hline Variables & Beta & Beta & Beta & Beta \\
Sex & 0.518 & 0.672 & 0.719 & 0.701 \\
Negative rumination (Time1) & $0.730^{*}$ & $0.617^{*}$ & $0.622^{*}$ & $0.624^{*}$ \\
Personal standards & & 0.060 & 0.052 & 0.110 \\
Thought suppresion & & $0.148^{+}$ & $0.142^{+}$ & $0.130^{+}$ \\
Stressor & & $0.116^{*}$ & $0.111^{*}$ & $0.113^{*}$ \\
Personal standards $\times$ Thought suppression & & & $0.021^{+}$ & 0.016 \\
Stressor $\times$ Thought suppression & & & $0.007^{+}$ & $0.009^{*}$ \\
Personal standards $\times$ Stressor & & & 0.000 & 0.001 \\
Personal standards $\times$ Sresssor $\times$ Thought suppression & & & $-0.002^{*}$ \\
Intercept & $31.173^{*}$ & $31.177^{*}$ & $30.927^{*}$ & $30.914^{*}$ \\
$F$-value & $180.892^{*}$ & $87.448^{*}$ & $56.190^{*}$ & $50.927^{*}$ \\
$\triangle R^{2}$ & $0.574^{*}$ & $0.048^{*}$ & 0.009 & $0.005^{*}$ \\
$R^{2}$ & $0.574^{*}$ & $0.622^{*}$ & $0.631^{*}$ & $0.636^{*}$ \\
\hline
\end{tabular}

Note. ${ }^{*} p<0.05,+p<0.10$.

Tests for a simple effect were performed for PS; for those who gained a high PS score, stressors contributed a significant positive association with negative rumination (difference in score from T1 to T2) at both high levels of thought suppression (simple effect: $\beta=0.110, t=2.34, p<0.05$ ) and low levels of thought suppression (simple effect: $\beta=0.126, t=3.14, p<0.05$ ). For those who attained a low PS score, stressors contributed a significant positive association, with negative rumination at high levels of thought suppression (simple effect: $\beta=0.201, t=4.21, p<0.05)$, but not at low levels of thought suppression $(\beta=0.016, t=0.34, p$ $=0.74)$. The results are depicted in Figure 1 .
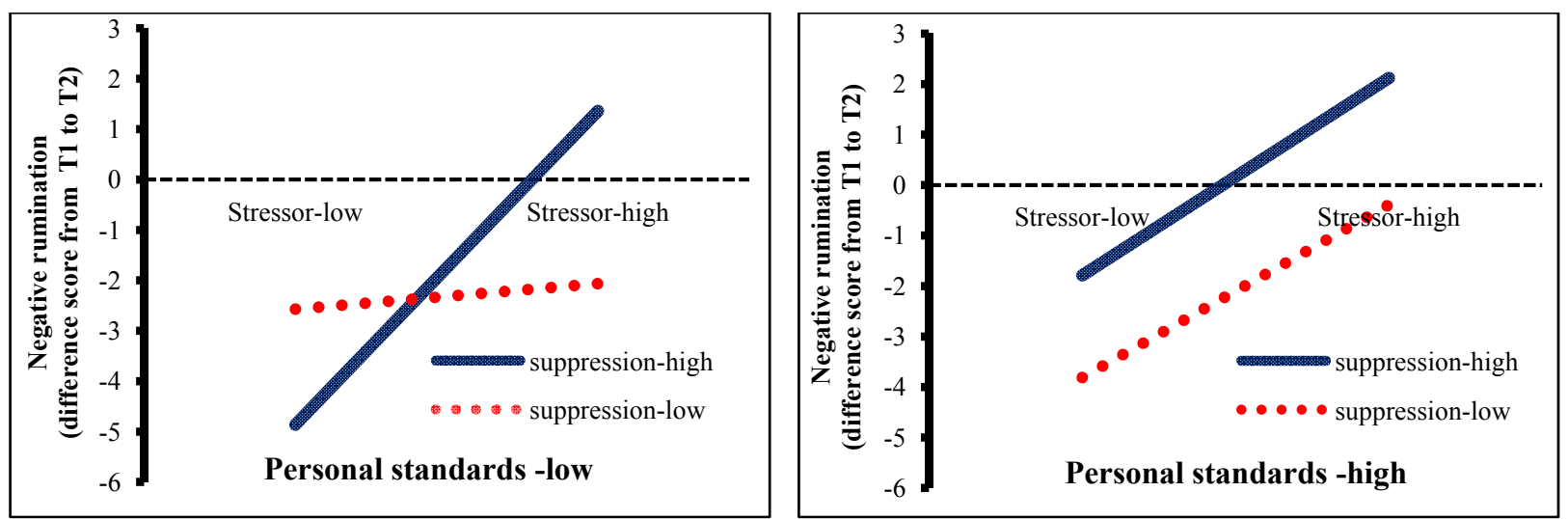

Figure 1. The interaction effect of personal standards (aspect of perfectionism), thought suppression, and stressor on negative rumination (difference score from T1 to T2) (Notes. high $=+1 S D$, low = -1 SD, T1 (Time-1), T2 (Time-2)).

\section{CM (Concern Over Mistakes)}

The results of the hierarchical multiple regression analysis relating to $\mathrm{CM}$ indicateda significant main effect for stressors $(\beta=0.111, p<0.05)$, a two-way interaction effect with thought suppression and CM 
( $\beta=0.032, p<0.05$ ), but a three-way interaction effect was not significant (see Table 3). Consideration of two-way interactions showed that those who gained a low CM score with high levels of thought suppression (simple effect: $\beta=0.202, t=2.24, p<0.05$ ), contributed a significant positive association with negative rumination.

Table 3

Hierarchical Multiple Regression Analysis of Negative Rumination (Time 2) on CM, Thought Suppresion, and Stressor

\begin{tabular}{lcccc}
\hline & Step 1 & Step 2 & Step 3 & Step 4 \\
\hline Variables & Beta & Beta & Beta & Beta \\
Sex & 0.518 & 0.607 & 0.549 & 0.558 \\
Negative rumination (Time 1) & $0.730^{*}$ & $0.600^{*}$ & $0.595^{*}$ & $0.595^{*}$ \\
Concern over mistakes & & 0.086 & 0.099 & 0.121 \\
Thought suppresion & & $0.145^{+}$ & $0.129^{+}$ & $0.145^{+}$ \\
Stressor & & $0.112^{*}$ & $0.108^{*}$ & $0.111^{*}$ \\
Concern over mistakes $\times$ Thought suppression & & & $0.032^{*}$ & $0.032^{*}$ \\
Stressor $\times$ Thought suppression & & & 0.004 & 0.005 \\
Concern over mistakes $\times$ Stressor & & & -0.001 & -0.002 \\
Concern over mistakes $\times$ Sresssor $\times$ Thought suppression & & & -0.001 \\
Intercept & $31.173^{*}$ & $31.175^{*}$ & $30.808^{*}$ & $30.826^{*}$ \\
$F$-value & $180.892^{*}$ & $87.535^{*}$ & $56.648^{*}$ & $50.927^{*}$ \\
$\triangle R^{2}$ & $0.574^{*}$ & $0.048^{*}$ & 0.011 & 0.001 \\
$R^{2}$ & $0.574^{*}$ & $0.622^{*}$ & $0.633^{*}$ & $0.634^{*}$ \\
\hline
\end{tabular}

Note. ${ }^{*} p<0.05,+p<0.10$.

\section{Discussion}

In this study, we attempted to clarify the mechanism through which a perfectionist would increase his or her intensity of negative rumination, by revealing the types of situation he/she might act on and the coping style he/she might engage in. The formation of a negative loop of thought has a strong relationship not only to injuring personal mental health but also to the possibility of a higher risk for depression onset and recurrence. Therefore, it is crucial for us to elucidate the possible factors that result in depressed rumination, and thereby devise effective preventable strategies for healthy living.

As shown in Table 2 and Table 3, perfectionists with high levels of PS show a significant relationship between coping and stressful situations. In the case of individuals who set higher goals (the right side of Figure1), negative rumination scores increase as the stressfulness of situations encountered increases, regardless of whether strange thought is attempted. In detail: If individuals suppress strange thoughts through great effort (high suppression), negative rumination increased from T1 to T2 as the situation faced became more stressful. The findings suggest that paradoxical effects of thought suppression result in one's coping strategy having the opposite effect to that designed to achieve (Wegner, 1989; Wegner et al., 1987). In the same way, individuals who are not eager to suppress strange thought (low suppression) gained an increase in negative rumination as the relevant stressor became larger. However, the negative rumination score for suppression-high individuals exceeds the 0-point when he/she confronts the stress situation with trying to suppress his or her thought. On the other hand, the negative rumination score for suppression-low individuals does not exceed the 
0-point when he/she does not rely on thought suppression. This result is impressive because it shows an interesting difference between suppression-high and -low individuals. Namely, suppression-high individuals show that aggravation leads to the onset and recurrence of depression, and suppression-low individuals can manage their own psychological distress without making excessive effort to do so. In this way, when perfectionists who set a higher aim are confronted with a severe situation, they try to suppress their own strange thoughts, and this effort acts as a casual factor for an increase in negative rumination.

For the non-perfectionist who does not necessarily set a higher aim (the left side of Figure 1), if he/she is coping with thought suppression for greater degrees of stress (suppression-high), negative rumination grows in a score between T1 and T2 if the situation becomes more stressful. However, individuals not suppressing their thought (suppression-low) do not show an increasing tendency toward negative rumination in accordance with the growth of the stressor. This suggests that suppression-low individuals are more invulnerable to the stressor than suppression-high individuals.

However, it is questionable as to whether perfectionists and non-perfectionists adopt the same strategy with regard to thought suppression in daily life. Perfectionists tend to attend to their own failures and mistakes due to their setting higher aims for themselves. Perhaps this behavior of setting a high goal brings mental and physical fatigue, while the perfectionist continues putting in effort with a possibility of "no reward". We may call this "the compulsive style" because perfectionists will continue to confront an endless number of stressors. On the other hand, it is hard to imagine that non-perfectionists would continue an endless effort, because they do not set such a high aim for themselves. Rather, they may regret their later predicament-a situation resulting from an unprudential escape from an aversive situation - without engaging in compulsive striving.

Actually, perfectionists and non-perfectionists may have quite different personality traits and thought suppression manners. If we consider these cognitive differences, especially the differences in the manner of thought suppression, we may be better able to apply the instruction to perfectionists as "try not to suppress your thoughts" rather than as "try to change your negative thoughts". This has implications for preventing the negative rumination loops shown among perfectionistic individuals.

It would be difficult for perfectionists not to suppress their own thoughts, because it is necessary for not suppressing thought that one be able to adopt mental distance from his or her intention to control thought. In such a case, mindfulness-based stress reduction (Kabat-Zinn, 1990) or Morita therapy (developed in Japan, see Kitanishi, 2001) may be useful, through their advocating "acceptance of oneself" as effective treatments in psychotherapy. The procedure of mindfulness, usually taught through meditation training, consists of learning to intentionally turn one's attention toward internal and external experiences occurring in the present moment (Kabat-Zinn, 1990). Through such practice, we may learn a method to adopt mental distance from negative thoughts. Deyo, Wilson, Ong, and Koopman (2009) demonstrated that mindfulness training for eight weeks among a non-clinical community sample increases an attitude of mindfulness and feelings of well-being and decreases rumination and symptoms of depression.

According to Morita therapy, excessive attention paid to one's own symptoms aggravates depression and anxiety. The symptom that results from this phenomenon is called "toraware" (Gibson, 1974). "Toraware" has been conceived to be evoked from endless efforts to not think about the symptom called "hakarai" (which is similar to the concept of thought suppression). Therefore, when directing clients to concentrate on the real act or behavior that he/she desires to perform, the client learns to take mental distance from the intention to control his or her thought suppression (Kitanishi, 2001). 
In summary, findings of the present study are as follows: When perfectionists are engaged in stressful situations in a compulsive manner, they ironically achieve a result opposite to what they originally sought, due to their compulsive way of enthusiastically engaging in thought suppression. In this case, one effective treatment for perfectionists might be if they could achieve freedom from the endless task of attempting to suppress their negative thoughts, as with the following instruction: "try not to suppress your own thoughts". This issue still remains an important problem and future detailed studies may be needed.

\section{References}

Aiken, L. S., \& West, S. G. (1991). Multiple regression: Testing and interpreting interactions. Thousand Oaks, C.A.: Sage.

Aldao, A., Nolen-Hoeksema, S., \& Schweizer, S. (2010). Emotion regulation strategies across psychopathology: A meta-analysis review. Clinical Psychological Review, 30, 217-237.

Cohen, J., \& Cohen, P. (1983). Applied multiple regression/correlation analysis for the behavioral sciences (2nd ed.). Hillsdale, N.J.: Erlbaum.

Deyo, M., Wilson, K., Ong, J., \& Koopman, C. (2009). Mindfulness and rumination: Does mindfulness training lead to reduction in the ruminative thinking associated with depression? The Journal of Science and Healing, 5, 265-271.

Enns, M. W., \& Cox, B. J. (2002). Nature and assessment of perfectionism. In G. L. Flett, \& P. L. Hewitt (Eds.), Perfectionism: Theory, research, and treatment. Washington, D.C.: American Psychological Association.

Gibson, H. B. (1974). Morita therapy and behavior therapy. Behavior Research and Therapy, 12, 347-353.

Hamachek, D. E. (1978). Psychodynamics of normal and neurotic perfectionism. Psychology: A Journal of Human Behavior, 15, 27-33.

Hewitt, P. L., \& Flett, G. L. (1991). Perfectionism in the self and social contexts: Conceptualization, assessment, and association with psychopathology. Journal of Personality and Social Psychology, 60, 456-470.

Ito, T., Takenaka, K., \& Agari, I. (2001). Study for psychological factors related to depression: A comparison between negative rumination, perfectionism, melancholic personality, and attributional style. Japanese Journal of Health Psychology, 14, $11-23$.

Kabat-Zinn, J. (1990). Full catastrophe living. The Bantam Dell Publishing.

Kitanishi, O. (2001). Pathology of self-obsession. Hakuyosha, Ltd.

Matsumoto, M. (2008). Development of expanded response styles questionnaire. The Japanese Journal of Personality, 16, 209-219.

Minarik, M. L., \& Ahrens, A. H. (1996). Relations of eating and symptoms of depression and anxiety to the dimensions of perfectionism among undergraduate women. Cognitive Therapy and Research, 20, 155-169.

Nolen-Hoeksema, S. (1991). Responses to depression and their effects on the duration of depressive episodes. Journal of Abnormal Psychology, 100, 569-582.

Kobori, O., \& Tanno, Y. (2004). Development of multidimensional perfectionism cognition inventory. Japanese Journal of Personality, 13, 34-43.

Schiena, R. S., Lumine, O., Philippot, P., \& Douilliez, C. (2012). Adaptive and maladaptive perfectionism in depression: Preliminary evidence on the role of adaptive and maladaptive rumination. Personality and Individual Differences, 53, 774-778.

Shima, N. (1992). The effects of social support on daily life stress in students. Japanese Journal of Social Psychology, 7, 45-53.

Shima, N. (1999). Development of stressor scale of daily hassles for undergraduate students. Chukyo University Department of Sociology Bulletin, 14, 69-83.

Wegner, D. M. (1989). White Bears and other unwanted thoughts. Advance Experimental Social Psychology, 25, 195-225.

Wegner, D. M., Schneider, D. J., Carter, S. R., \& White, T. L. (1987). Paradoxical effects of thought suppression. Journal of Personality and Social Psychology, 53, 5-13.

Wegner, D. M., \& Zanakos, S. (1994). Chronic thought suppression. Journal of Personality, 62, 615-640. 